\title{
Experimental observation of oblique shock waves in steady non-ideal flows
}

\author{
Marta Zocca ${ }^{1} \cdot$ Alberto Guardone $^{1} \mathbb{1} \cdot$ Giorgia Cammi $^{2} \cdot$ Fabio Cozzi $^{2} \cdot$ Andrea Spinelli $^{2}$
}

Received: 3 April 2019 / Accepted: 3 May 2019 / Published online: 27 May 2019

(C) The Author(s) 2019

\begin{abstract}
Steady oblique shock waves are observed for the first time in non-ideal supersonic flows of single-phase organic vapors. A diamond-shaped airfoil with semi-aperture $7.5^{\circ}$ at the leading edge and $10^{\circ}$ at the trailing edge is placed within a uniform supersonic stream (Mach number $M=1.5$ ) of siloxane fluid MDM (octamethyltrisiloxane, $\mathrm{C}_{8} \mathrm{H}_{24} \mathrm{O}_{2} \mathrm{Si}_{3}$ ). Oblique shock waves are observed at varying stagnation conditions in the pre-shock state, for a set of flow deviation angles obtained by changing the attitude of the model with respect to the wind tunnel axis. Stagnation temperatures and pressures reduced by critical values range from 0.928 to 0.979 and from 0.200 to 0.615 , respectively. Oblique shock waves are characterized through independent measurements of pressure, temperature and Mach number. Stagnation pressure and temperature are measured in the wind-tunnel settling chamber, while the pre-shock, post-shock and post-expansion static pressures are measured at selected locations close to the airfoil. Direct measurement of the flow Mach number is provided by schlieren visualizations. Experimental results agree with the shock wave theory for two-dimensional steady flows, complemented with state-of-the-art thermodynamic models, and deviate from their well-known, dilute-gas predictions. In particular, measurements show the non-ideal dependence of the pressure ratio across the shock on stagnation conditions in addition to the well-known dependence on the pre-shock Mach number, specific heat ratio and flow deviation angle typical of dilute gas conditions.
\end{abstract}

\section{Graphical abstract}

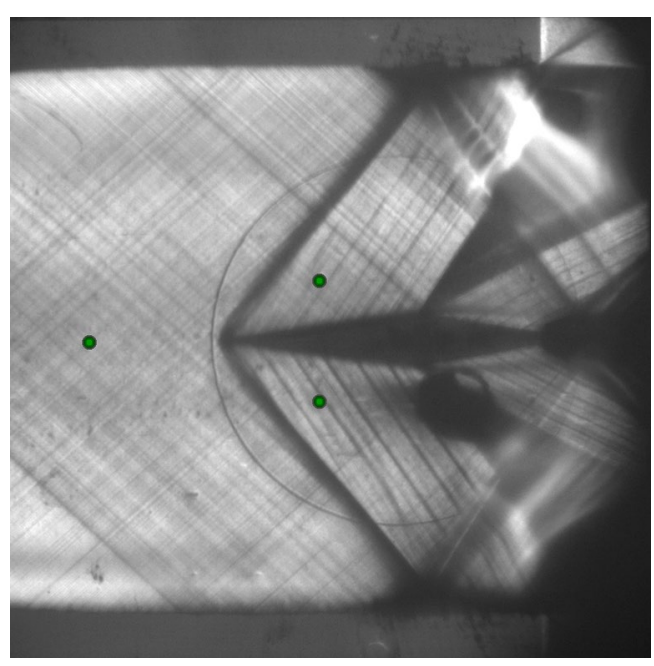

Alberto Guardone

alberto.guardone@polimi.it

Extended author information available on the last page of the article

\section{Introduction}

The knowledge of the flow behavior in aerodynamic devices operating in dilute-gas conditions is well established and satisfactory. On the contrary, scarce measurements are 
available for fluid flows featuring so-called non-ideal behavior close to saturation and critical point, or within the supercritical region. These so-called non-ideal compressible-fluid flows are encountered in a number of technical applications, including wind tunnels for aerodynamic testing and turbomachinery. In process engineering applications, non-ideal flow conditions are found, for example, in Rapid Expansion of Supercritical Solutions (RESS) of $\mathrm{CO}_{2}$, see Reverchon and Pallado (1996), Subramaniam et al. (1997), Sun et al. (2002) and reference therein, supercritical $\mathrm{CO}_{2}$ power systems (Angelino 1969; Feher 1968) and Organic Rankine Cycle (ORC) plants, namely Rankine cycles using a molecularly complex organic compound instead of steam as working fluid (see e.g. Angelino et al. 1991; Larjola 1995; Colonna et al. 2015; Macchi and Astolfi 2016). Rankine cycles operating with organic fluids are particularly suitable for the exploitation of heat sources such as waste heat from industrial processes, geothermal reservoirs, biomass combustion, and concentrated solar radiation. The thermodynamic state of the single-phase vapor at the inlet of an ORC turbine stator usually belongs to the non-ideal thermodynamic region. Moreover, due to high molecular complexity, high molecular mass, and non-ideal effects, the speed of sound in these flows is low compared to that of air and other simple gases, and expansion processes in turbine stators likely involve supersonic outlet flows even if modest flow velocities are attained, see Colonna and Guardone (2006), Colonna et al. (2008). In these conditions, oblique shock waves occur within the blade passages and at the trailing edge of the blades, thus entailing significant entropy generation (Denton 1993). In this respect, an accurate treatment of oblique compression and expansion waves in the non-ideal regime is key for a proper design of aerodynamic devices performing compression and expansion of flows which can be no longer described by the dilute-gas model.

The branch of fluid mechanics devoted to the study of flows in non-ideal conditions is termed Non-Ideal Compressible-Fluid Dynamics (NICFD). Here, the term non-ideal does not refer to dissipative mechanisms such as internal friction and heat conduction or to chemical reactions. Rather, it indicates the occurrence of peculiar flow behavior because of departure from dilute, ideal-gas thermodynamics. In NICFD, it is customary to distinguish between the so-called nonclassical $(\Gamma \leq 0)$ and classical $(\Gamma>0)$ regimes. The thermodynamic quantity $\Gamma$, where $\Gamma=1+(\rho / c)(\partial c / \partial \rho)_{s}$, with $\rho, c$ and $s$ as density, speed of sound and specific entropy per unit mass, is the so-called fundamental derivative of gasdynamics introduced by Thompson (1971). In the classical regime, admissible shock waves are of the compressive type only and rarefaction waves are associated with smooth, isentropic flows. In the non-classical regime, instead, peculiar flow features such as rarefaction shocks are physically admissible, i.e. result from the dynamic evolution of the flow towards thermodynamically stable states at higher entropy (Zamfirescu et al. 2008; Guardone et al. 2009). A further distinction can be made within the classical regime: for $\Gamma>1$, the speed of sound $c$ increases with pressure $P$, which is the typical behavior of dilute gases. Indeed, for a Polytropic (i.e. constant-specific-heat) Ideal Gas (PIG), one has $\Gamma=(\gamma+1) / 2$, which is always positive and greater than one. For $0<\Gamma<1$, which is referred to as non-ideal classical regime, the speed of sound decreases with pressure, see for instance Lambrakis and Thompson (1972) and Kluwick (2017). Relevant phenomena occurring in the non-ideal classical regime include the decrease of the flow Mach number along supersonic expansions (Cramer and Best 1991), the non-ideal evolution of the flow Mach number in diabatic supersonic nozzle flows (Schnerr and Leidner 1991) and the increase of the flow Mach number across oblique shocks of the compressive type (Vimercati et al. 2018). This latter phenomenon is ascribable to the non-monotone variation of the speed of sound along the shock adiabat, which is possible only for $\Gamma<1$.

The present work focuses on supersonic flows of siloxane compound MDM (octamethyltrisiloxane, $\mathrm{C}_{8} \mathrm{H}_{24} \mathrm{O}_{2} \mathrm{Si}_{3}$, see Table 1) in the non-ideal classical regime. Experiments are carried out within the TROVA supersonic wind tunnel at Politecnico di Milano, see Spinelli et al. (2013), Guardone et al. (2013), Spinelli et al. (2017) and Spinelli et al. (2018). In the same facility, Spinelli et al. (2018) recently proved that for symmetric planar converging-diverging nozzles of fixed geometry operating in the non-ideal classical regime, flows at different exhaust Mach number and exhaust to total pressure ratios are attained at varying stagnation conditions upstream of the nozzle. As it is well known, the flow dynamics of isentropic, non-shocked flows depends only on the ratio of the reservoir to ambient pressure and on the (constant) specific heat ratio under the polytropic ideal gas assumptions. On the contrary, the dynamic evolution of socalled non-ideal flows depends on both the local and total thermodynamic states of the fluid. The experimental investigation presented in this work aims at characterizing oblique shock waves in the non-ideal classical regime through independent measurements of pressure, temperature and Mach number, and to prove that, in this particular regime, the postshock state depends on both the total and local pre-shock conditions. The present work also aims at providing a first experimental database to be exploited by the NICFD community for future assessment of thermodynamic models and CFD tools for NICFD flows.

The $T-s$ diagram in Fig. 1 reports the saturation dome of MDM and isolines of the fundamental derivative of gasdynamics $\Gamma$ in the range $0<\Gamma<1$, as predicted by the Span-Wagner (SW) Equation of State (EoS) (Thol et al. 2017). The $\Gamma$-isolines enclose the so-called non-ideal classical region, which is located in the single-phase vapor region 


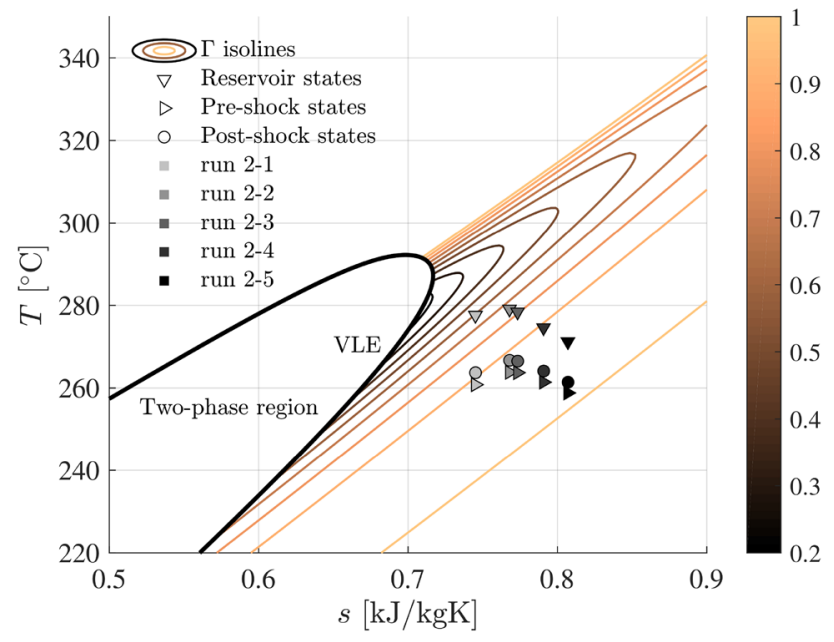

Fig. 1 Saturation dome of MDM and measured reservoir, pre-shock and post-shock states

Table 1 Properties of the working fluid of the TROVA wind tunnel

\begin{tabular}{ll}
\hline Short name & $\mathrm{MDM}$ \\
Full name & Octamethyltrisiloxane \\
Chemical formula & $\mathrm{C}_{8} \mathrm{H}_{24} \mathrm{O}_{2} \mathrm{Si}_{3}$ \\
Classification & Linear siloxane \\
Molecular weight $(\mathrm{kg} / \mathrm{kmol})$ & 236.53146 \\
Critical temperature $\left({ }^{\circ} \mathrm{C}\right)$ & 292.21 \\
Critical pressure $(\mathrm{bar})$ & 14.375 \\
Critical density $\left(\mathrm{mol} / \mathrm{dm}^{3}\right)$ & 1.134 \\
Normal boiling point temperature $\left({ }^{\circ} \mathrm{C}\right)$ & 152.48 \\
Reference & Thol et al. $(2017)$ \\
\hline
\end{tabular}

and displays the lowest values of $\Gamma$ in the close proximity of the saturated vapor curve and critical point. On the diagram, relevant reservoir, pre- and post-shock states obtained from a typical test (test 2) are reported. The corresponding datasets, labelled runs $2-1$ to runs $2-5$, where the second digit indicates ordering in time, are obtained at progressing testing time and are reported in Table 2 . The reported data clearly show that reservoir, pre-shock and post-shock conditions depart from dense-gas conditions and get close to dilute ones at progressing testing time, or, in other words, they are characterized by increasing values of $\Gamma$. Process conditions for the exemplary test displayed in Fig. 1 are chosen so as to attain the lowest possible value of $\Gamma$, (i.e. the most prominent depart from ideal conditions) in the pre-shock state. Specifically, as discussed by Spinelli et al. (2018), the reservoir condition upstream of the supersonic expansion in the wind-tunnel nozzle are set to achieve the lowest possible values of $\Gamma$ in the stagnation state, whereby not exceeding the thermal stability limit of the working fluid. Indeed, thermal stability issues pose severe limitations to the experimental observation of highly non-ideal supersonic flows, and limit the experimental investigation of non-ideal phenomena to mildly non-ideal and low-supersonic flows, see for example Keulen et al. (2018).

The present paper is structured as follows. Sections 2 and 3 present the experimental set-up and the fluid-dynamic and thermodynamic models used to investigate flows with shock waves and in the non-ideal classical regime, respectively. Results are discussed in Sect. 4 and comments upon the non-ideal nature of the oblique shock waves under scrutiny are provided. Concluding remarks are given in Sect. 5.

\section{Experimental set-up}

In this section, the experimental set-up is described. All the experiments reported in the present work were carried out in the Test-Rig for Organic VApours (TROVA) of the Laboratory of Compressible-fluid dynamics for Renewable Energy Applications (CREA) of Politecnico di Milano. The TROVA is a supersonic blow-down wind tunnel designed to investigate NICFD flows of a wide variety of organic fluids. In the experiments presented here, the wind tunnel is operated with the siloxane compound MDM (octamethyltrisiloxane $\mathrm{C}_{8} \mathrm{H}_{24} \mathrm{O}_{2} \mathrm{Si}_{3}$ ), which is liquid at room temperature and pressure, see Table 1. The TROVA operates in a discontinuous way to reduce the thermal power required for vapor production. The working fluid is discharged from a high-pressure vessel to a low-pressure vessel through the test section, which in the present campaign accommodates the model of a diamond-shaped airfoil. Details regarding the TROVA test rig, the design of the wind-tunnel de Laval nozzle, and the measurement techniques for the characterization of non-ideal supersonic flows can be found in Spinelli et al. (2013), Guardone et al. (2013), Cozzi et al. (2015) and Spinelli et al. (2018), respectively. A brief description of the test rig and instrumentation is reported here for completeness in Sect. 2.1. Section 2.2 focuses on the geometrical configuration of the test section for the observation oblique shock waves in the non-ideal regime.

\subsection{Test-rig and instrumentation}

In all tests, the TROVA is operated as a conventional blowdown supersonic wind tunnel. The wind-tunnel de Laval nozzle discharges a parallel uniform supersonic stream in a constant-area duct, where a diamond-shaped airfoil is placed. Figure 2a shows the installed test section, while Fig. $2 \mathrm{~b}$ shows the mirror-polished steel plate accommodating the planar nozzle and the diamond airfoil. Specifically, the nozzle is made of contoured profiles, which can be easily replaced whenever a different configuration needs to be tested. 


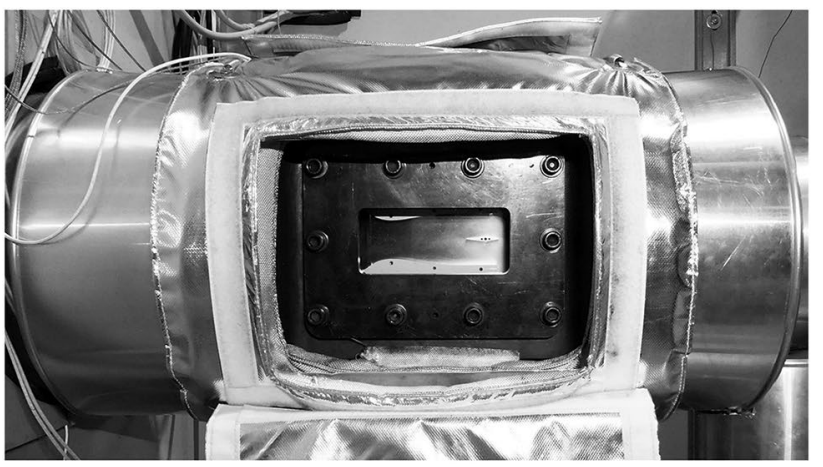

(a)

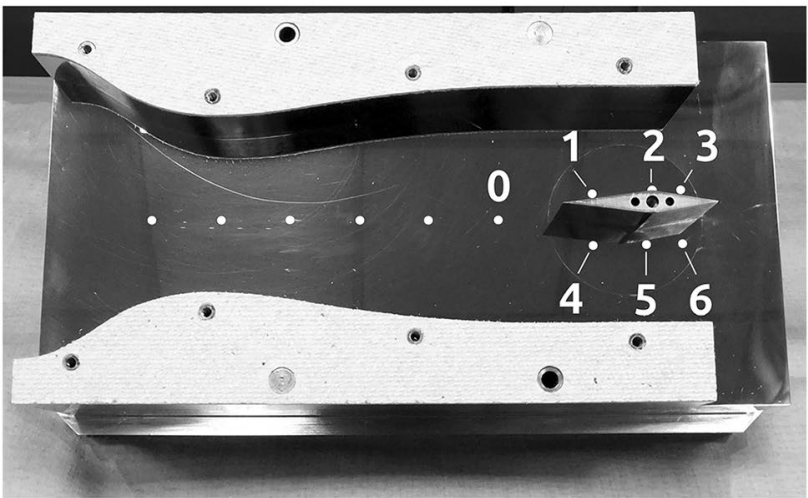

(b)

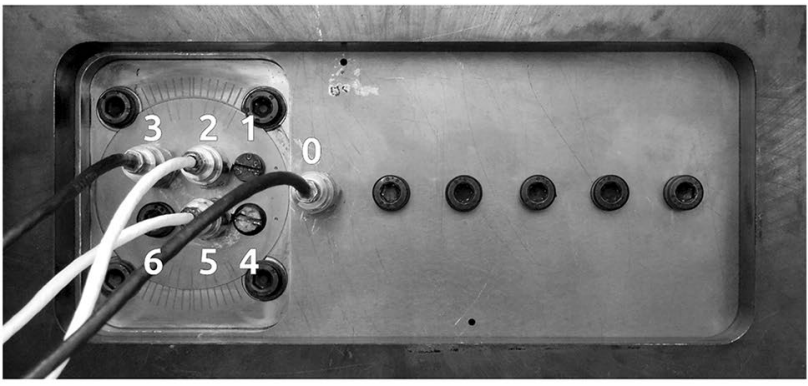

(c)

Fig. 2 a Test section of the TROVA. Flow direction is from left to right. b Mirror-polished back-closure of the test section with converging-diverging nozzle and diamond-shaped airfoil. Static pressure taps are highlighted: 0-6. c Back closure of the test section and view of the pressure transducers as mounted. Pressure taps 0, 2, 3 and 5 are connected to transducers (active taps)

Piezo-resistive pressure transducers (Kulite XTEH-7L series), with full scale in the range 3.5-40 bar and calibrated both in pressure and temperature in the range 1 bar-FS (full scale) and $25-300{ }^{\circ} \mathrm{C}$, are used to measure static pressures at discrete locations within the flow field, which are marked with white dots in Fig. 2b). Pressure transducers are mounted as illustrated in Fig. 2c. Due to the very low flow velocity in the settling chamber $(\sim 1 \mathrm{~m} / \mathrm{s})$, the same arrangement implemented for static pressure measurements is adopted for the measurement of the stagnation pressure. After calibration, an expanded uncertainty of $0.07 \%$ of the full scale for all transducers (95\% confidence interval) was obtained. Each pressure tap on the nozzle axis is connected to a transducer by a $30-\mathrm{mm}$-long line-cavity system, followed by a $130 \mathrm{~mm}^{3}$ capacity ahead of the sensing element. For the linecavity system, a resonance frequency of the order of $200 \mathrm{~Hz}$ was estimated according to the viscous model proposed by Antonini et al. (2008). To measure the stagnation temperature, the hot junctions of a J-type $(\mathrm{Fe}-\mathrm{Cu} / \mathrm{Ni})$ and of a K-type $(\mathrm{Ni} / \mathrm{Cr}-\mathrm{Ni} / \mathrm{Al})$ thermocouple are positioned at the symmetry axis of the settling chamber, at the same axial location of the total pressure tap and $200 \mathrm{~mm}$ upstream of it, respectively. The diameter of the hot junction is $0.7 \mathrm{~mm}$ for the J-type and $0.25 \mathrm{~mm}$ for the K-type thermocouple. The time constants of the J-type and the K-type thermocouples, estimated according to the method proposed by Hung et al. (2005), are $1.34 \mathrm{~s}$ and $0.28 \mathrm{~s}$, respectively. Calibration of thermocouples in the range $25-300^{\circ} \mathrm{C}$ results in expanded uncertainties of $1.2^{\circ} \mathrm{C}$ (J-type) and $1.0^{\circ} \mathrm{C}$ (K-type) on each measured temperature. Both pressure transducers and thermocouples provide voltage output signals, which are amplified by an analog signal conditioning system before being sampled and converted to digital ones by a high-speed data acquisition board. A software developed in LabView ${ }^{\circledR}$ environment manages the plant control and the data acquisition.

Schlieren visualizations of the flow within the test section complement pressure and temperature measurements. The front planar wall of the test section is a quartz window allowing for optical access to the flow, while the rear closure is a mirror-polished steel plate. Since optical access is guaranteed only from one side of the test section, a doublepassage parallel-light schlieren system is used instead of the classical Z-type configuration. The emitting and receiving optical components are mounted on the same bench, thus the optical path lies in a plane containing the nozzle symmetry axis and a line perpendicular to the lateral walls of the test section (i.e. the front quartz window and the back closure). Schlieren images are recorded for the entire duration of the test by a CMOS camera. At test start, a digital triggering signal is sent to the camera to synchronize the schlieren images with pressure and temperature readings. The camera records the schlieren images at a rate of $25 \mathrm{fps}$. Pressure and temperature data, acquired at a frequency of $1 \mathrm{kHz}$, are averaged in packages of 40 points to synch with schlieren images, resulting in a time resolution of $40 \mathrm{~ms}$ for the whole dataset. The image resolution is $1936 \times 1216 \mathrm{px}$, which gives $12.7 \mathrm{px} / \mathrm{mm}$, and the exposure time is $1 \mathrm{~ms}$. In all recorded images, the schlieren knife is rotated so that darker gray levels are associated with positive density gradients (compressions) and lighter gray levels to negative ones (expansions), in the flow direction. Details on the schlieren set-up of the TROVA can be found in Cozzi et al. (2015).

Besides providing a visualization of the whole flow field, schlieren images allow to perform a direct measurement of 
the local flow Mach number. In the work by Spinelli et al. (2018), the authors applied this technique to the measurement of the flow Mach number along the symmetry axis of planar converging-diverging nozzles operating with siloxane fluid MDM, in test conditions similar to the ones examined in the present experimental campaign. In the supersonic portion of the flow domain, schlieren visualizations display the Mach waves originating from the contoured profiles of the test section due to enhanced surface roughness. The line-detection algorithm proposed in Lo and Tsai (1995) is applied to the schlieren frames to determine the Mach angle $\mu$, which is related to the local Mach number by $M=1 / \sin \mu$. Specifically, the angle $\mu$ is the slope of the Mach waves with respect to the local flow direction. Along the nozzle axis, the local flow direction lies on the symmetry axis of the nozzle. On the airfoil, the local flow direction is imposed by the slope of its compression and expansion ramps. The expanded uncertainty, $U_{M}$, of the measured local Mach number (Gaussian, 95\% confidence level) is computed as

$U_{M}=M \sqrt{M^{2}-1} U_{\mu}$,

where $M$ is the Mach number and $U_{\mu}$ is the angular expanded uncertainty ( $95 \%$ confidence level), computed by assuming a uniform probability distribution within the pixel of the end point location. As expected, the uncertainty of Mach number measurements $U_{M}$ increases with the flow Mach number, due to the dependence of the Mach number on the slope of the Mach waves $\mu$ through the inverse sine function.

\subsection{Test section and model}

The geometry of the test section is shown in Fig. 3a in dimensional units. A planar converging-diverging nozzle is designed to discharge a parallel uniform supersonic stream towards a diamond-shaped airfoil. A schematic representation of the flow pattern around the airfoil in design conditions is given in Fig. 4. At the leading edge of the airfoil, the supersonic flow discharged by the nozzle is compressed and deflected by two oblique shock waves. At the shoulder, the flow is expanded by two centered expansion fans. At the trailing edge, the flow is again compressed and deflected by two oblique shocks.

In design conditions, the wind-tunnel de Laval nozzle produces an expansion from total conditions $P^{\mathrm{t}}=10 \mathrm{bar}$ and $T^{\mathrm{t}}=277^{\circ} \mathrm{C}$ to pressure $P_{\mathrm{e}}=3.21$ bar and exhaust Mach number $M_{\mathrm{e}}=1.5$. The profile of the diverging portion of the nozzle is designed by applying the method of characteristics according to the procedure described by Guardone et al. (2013). At the throat, located at $43 \mathrm{~mm}$ from inlet, the profiles of the converging and diverging portions match with horizontal tangent and continuous curvature. The converging

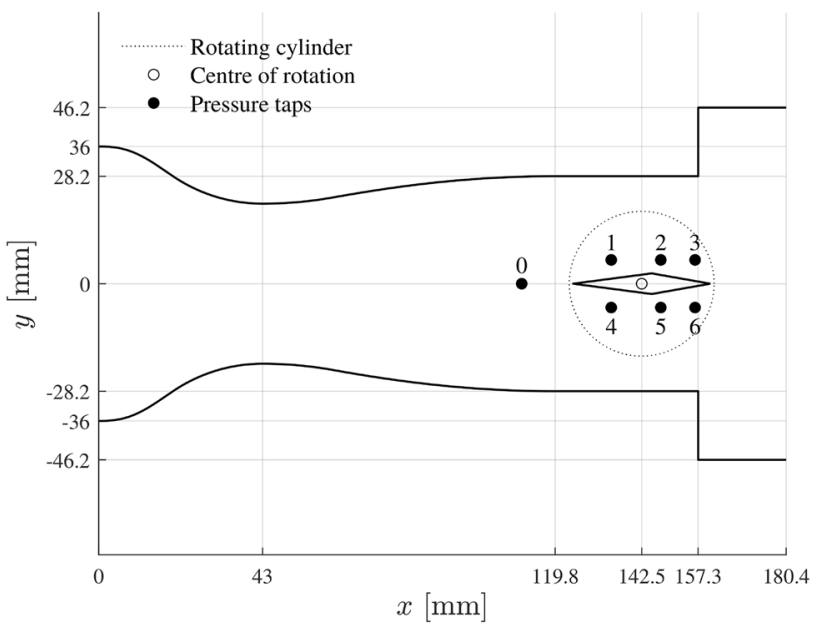

(a)

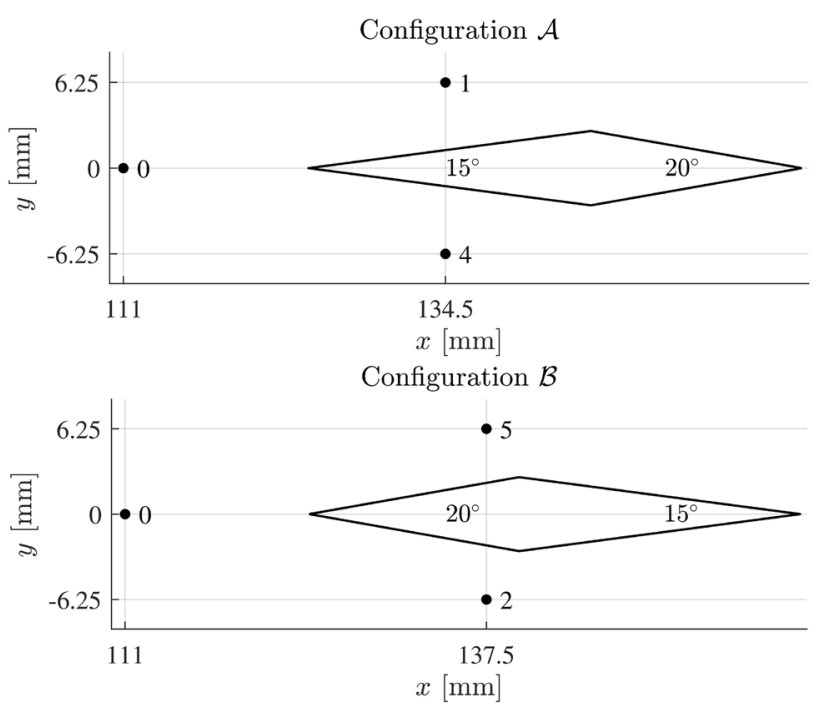

(b)

Fig. 3 a Geometry of the test section in dimensional units. b Geometry of the diamond airfoil and position of the active static pressure taps in configurations $\mathcal{A}$ and $\mathcal{B}$

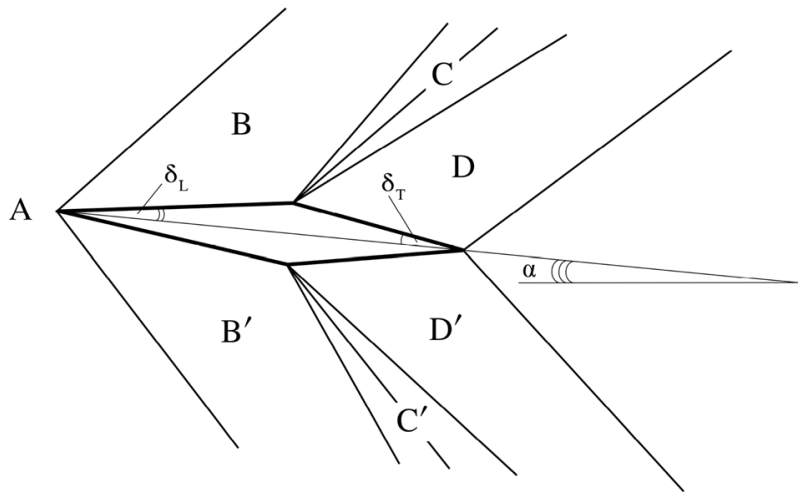

Fig. 4 Wave pattern for a uniform supersonic flow around a diamondshaped airfoil. $\delta_{\mathrm{L}}$ and $\delta_{\mathrm{T}}$ are the semi-aperture at the leading and trailing edge, respectively 
portion is a fifth-order polynomial curve, matched smoothly with a circular arc of radius $42 \mathrm{~mm}$ at the throat, i.e. twice the throat half-height $(21 \mathrm{~mm})$.

Note that, due to the non-ideal flow dependence on the stagnation conditions, the nozzle operates in design conditions only over a limited interval of time or, equivalently, reservoir conditions. Also, during the experimental runs, only a limited portion of the flow in the close proximity of the nozzle walls, and hence away from the airfoil, was observed to depart from uniform flow conditions. Therefore, the flow is assumed to be uniform and parallel to the nozzle axis at the nozzle exit in the following.

The nozzle discharges the flow into a 37.5-mm-long constant-area duct, where the airfoil is placed. The duct ends at $157.3 \mathrm{~mm}$ from inlet and eventually discharges into the downstream ambient. The airfoil, of chord $c=36 \mathrm{~mm}$, is cantilever mounted on a rotating cylinder, whose rotation allows to adjust the attitude of the airfoil with respect to the horizontal axis of the test section with a resolution of $1^{\circ}$. A detail view of the airfoil is reported in Fig. 3b. In configuration $\mathcal{A}$, the semi-apertures at the leading and trailing edge are $\delta_{\mathrm{L}}=7.5^{\circ}$ and $\delta_{\mathrm{T}}=10^{\circ}$, respectively. In configuration $\mathcal{B}$, $\delta_{\mathrm{L}}=10^{\circ}$ and $\delta_{\mathrm{T}}=7.5^{\circ}$. The position of the rotating cylinder is sketched in Fig. 3a. A $180^{\circ}$ rotation of the cylinder allows to switch from configuration $\mathcal{A}$ to configuration $\mathcal{B}$.

Pressure measurement points are marked with black dots in Fig. 3a. The static pressure in the flow upstream of the airfoil is measured at the nozzle axis, through the pressure tap identified as 0 . Pressure taps $1-6$ are machined in the rotating cylinder, and rotate with the airfoil at changing attitude. In each of the two possible configurations, $\mathcal{A}$ and $\mathcal{B}$, two pressure taps around the airfoil, are used. The active pressure taps for each configuration are reported in Fig. 3b: 1 and 4 in configuration $\mathcal{A}, 2$ and 5 in configuration $\mathcal{B}$.

\section{Operating conditions and data representation}

The batch operating mode of the TROVA is such that, at each time instant during the test, a different dataset including total temperature, total and static pressures and schlieren images is acquired. Since the characteristic time of the emptying of the high-pressure reservoir $\Delta t_{\text {reservoir }}$ is more than two orders of magnitude greater than the flow residence time in the de Laval nozzle $\Delta t_{\text {nozzle }}$ (see Spinelli et al. 2013) and the latter, in turn, satisfies $\Delta t_{\text {nozzle }} \ll \tau$, where $\tau$ is the thermodynamic relaxation time (see e.g. Thompson 1988; Colonna et al. 2007), each dataset is considered here as a steady-state representation of the flow field. Throughout the paper, datasets are labelled as runs $i-j$, where $i$ identifies the test run and $j$ the flow conditions attained at different time instants during the test.
At the design point, the wind-tunnel nozzle discharges a uniform supersonic flow at $M=1.5$, whose thermodynamic state is defined by $P=3.21$ bar and $T=258^{\circ} \mathrm{C}$. In terms of reduced variables made dimensionless by critical point values, the thermodynamic state upstream of the airfoil is $P_{r}=P / P_{c}=0.223$ and $T_{r}=T / T_{c}=0.939$, where $P_{c}=14.375$ bar and $T_{c}=292.2^{\circ} \mathrm{C}$ are the critical pressure and temperature of MDM. The corresponding value of the fundamental derivative of gasdynamics is $\Gamma=0.901$, and the compressibility factor $Z=P v / R T$ is 0.892 , where $R=\mathcal{R} / \mathcal{M}$ is the gas constant, with $\mathcal{R}$ universal gas constant and $\mathcal{M}$ molecular weight. Note that for a dilute gas $P v \equiv R T$ and the compressibility factor is identically one.

In each test, the active pressure taps are placed in the three uniform flow regions identified as $\mathrm{A}, \mathrm{B}$, and $\mathrm{B}^{\prime}$ in Fig. 4. Specifically, pre-shock pressure $P_{\mathrm{A}}$ is measured at tap 0 , and post-shock pressures $P_{\mathrm{B}}$ and $P_{\mathrm{B}^{\prime}}$ are measured at taps 1 and 4 in configuration $\mathcal{A}$ (5 and 2 in configuration $\mathcal{B}$ ).

Throughout the paper, measurements are compared to the shock wave theory (see Thompson 1988 for reference), complemented with the multiparameter Span-Wagner (SW) EoS by Thol et al. (2017). To compute the post-shock conditions according to the shock wave theory, the specific total enthalpy $h^{\mathrm{t}}=h+\frac{1}{2} u^{2}$, with $h$ enthalpy and $u$ velocity module, respectively, and the entropy $s$ assumed to be constant along the expansion in the nozzle. Moreover, since the kinetic energy contribution can be assumed to be negligible in the settling chamber $\mathrm{R}\left(\frac{1}{2} u_{\mathrm{R}}^{2} \ll h_{\mathrm{R}}\right)$, then $h^{\mathrm{t}} \simeq h_{\mathrm{R}}$ and $s \simeq s_{\mathrm{R}}$. The enthalpy and entropy in the settling chamber are computed using the SW model as $h_{\mathrm{R}}=h\left(P_{\mathrm{R}}, T_{\mathrm{R}}\right)$ and $s_{\mathrm{R}}=s\left(P_{\mathrm{R}}, T_{\mathrm{R}}\right)$ from the measured values of the pressure $P_{\mathrm{R}}$ and the temperature $T_{\mathrm{R}}$. The thermodynamic state of the flow in region $\mathrm{A}$ is, therefore, computed from the measured pressure $P_{\mathrm{A}}$ (measured at pressure tap 0 ) and the calculated value of the entropy $s_{R}$. The flow velocity in region $\mathrm{A}$ is determined by enforcing the energy conservation law and assuming uniform parallel velocity, namely $u_{\mathrm{A}}=\sqrt{2\left[h_{\mathrm{R}}-h\left(P_{\mathrm{A}}, s_{\mathrm{R}}\right)\right]}$ at the exit of the nozzle.

With reference to Fig. 4, the thermodynamic state and the flow velocity in region A together define the pre-shock state $\left(P_{\mathrm{A}}, v_{\mathrm{A}}, \mathbf{u}_{\mathrm{A}}\right)$. The post-shock state $\left(P_{\mathrm{B}}, v_{\mathrm{B}}, \mathbf{u}_{\mathrm{B}}\right)$ is obtained from the well-known Rankine-Hugoniot jump relations complemented by the SW EoS by imposing the flow deviation angle $\vartheta_{\mathrm{B}}=\delta_{\mathrm{L}}-\alpha$ at the compression ramp on the top (bottom) surface of the airfoil. The shock angle $\beta$ is also obtained.

\section{Results and discussion}

Measured data are now presented and compared to results from the shock wave theory. For each test, five datasets including $P_{\mathrm{R}}, T_{\mathrm{R}}, P_{\mathrm{A}}$, and $P_{\mathrm{B}}$, and the corresponding schlieren images are provided. Each dataset describes a 
Fig. 5 Schlieren visualizations. a Configuration $\mathcal{A}, \quad \alpha=0^{\circ}$, $\Gamma_{\mathrm{A}}=0.917, \mathbf{b}$ configuration $B, \alpha=0^{\circ}, \Gamma_{\mathrm{A}}=0.942$, c configuration $\mathcal{B}, \alpha=1^{\circ}, \Gamma_{\mathrm{A}}=0.936$. Active pressure taps are marked with a dot in $\mathbf{a}$ and $\mathbf{b}$

steady-state flow arrangement obtained at increasing value of the pre-shock $\Gamma$, for which pre-shock, post-shock and post-expansion states can be determined following the procedure described in the previous paragraph. For the $i$ th test run, the five datasets are identified by the calculated value of the pre-shock fundamental derivative $\Gamma_{\mathrm{A}}$ and are labelled progressively as run $i-1$, run $i-2$, run $i-3$, run $i-4$, and run $i-5$.

Tests are run in configuration $\mathcal{A}$ at attitudes $\alpha=0^{\circ}, 1^{\circ}$, and in configuration $\mathcal{B}$ at attitudes $\alpha=0^{\circ}, 1^{\circ}, 2^{\circ}$, giving the following set of deviation angles for the compression ramps: $\vartheta_{\mathrm{B}}=6.5^{\circ}, 7.5^{\circ}, 8^{\circ}, 8.5^{\circ}, 9^{\circ}, 10^{\circ}, 11^{\circ}, 12^{\circ}$.

Section 4.1 describes the flow layout as observed from schlieren visualizations, while results of pressure, temperature, Mach number and shock angle measurements are summarized in Sect. 4.2. A discussion of non-ideal effects based on the experimental results of Sect. 4.2 is carried out in Sect. 4.3.

\subsection{Flow layout from schlieren visualizations}

Schlieren visualizations revealed the occurrence of three different flow patterns at varying airfoil attitude and configuration. Figure 5 reports schlieren visualizations of the flow field in configuration $\mathcal{A}, \alpha=0^{\circ}$ (Fig. 5a), in configuration $\mathcal{B}, \alpha=0^{\circ}$ (Fig. 5b), and in configuration $\mathcal{B}, \alpha=1^{\circ}$ (Fig. 5c). The three frames are associated with pre-shock fundamental derivatives $\Gamma_{\mathrm{A}}=0.917, \Gamma_{\mathrm{A}}=0.942$, and $\Gamma_{\mathrm{A}}=0.936$, respectively. In Fig. 5a, b, the flow pattern closely resembles the one expected from the inviscid shock-expansion theory: two oblique shocks form at the airfoil leading edge and two centered expansion fans form at the shoulder. The flow deviation angles imposed by the compression ramps are $\vartheta_{\mathrm{B}}=7.5^{\circ}$ and $\vartheta_{\mathrm{B}}=10^{\circ}$, respectively. In Fig. 5c, the wave pattern on the bottom surface of the airfoil is qualitatively different from the expected one, possibly due to blockage effects or to flow conditions exceeding the maximum deviation angle for the admissibility of attached oblique shocks. In the configuration depicted in Fig. 5c, the flow deviation angles imposed by the compression ramps are $\vartheta_{\mathrm{B}}=9^{\circ}$ on the top surface and $\vartheta_{\mathrm{B}^{\prime}}=11^{\circ}$ on the bottom surface of the airfoil. In all the tested configurations, compression ramps deflecting the flow through angles less than or equal to $10^{\circ}$ originate oblique leading edge shocks closely matching the ones expected from the shock-expansion theory. For flow deviation angles $\vartheta_{\mathrm{B}}>10^{\circ}$, the flow layout is qualitatively similar to the one observed on the bottom surface of the airfoil in Fig. 5c and, therefore, the corresponding measurements are neglected in the following analysis. The flow visualizations of Fig. 5a, b

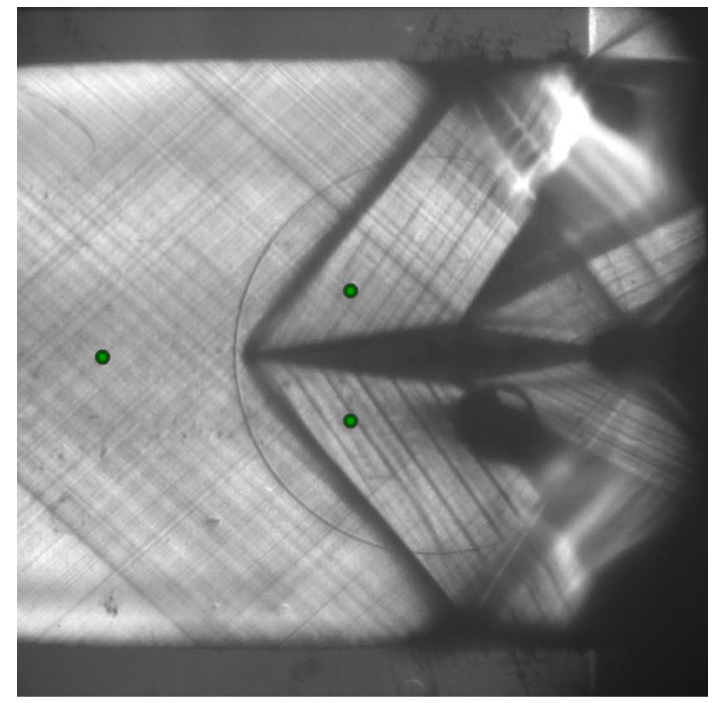

(a)

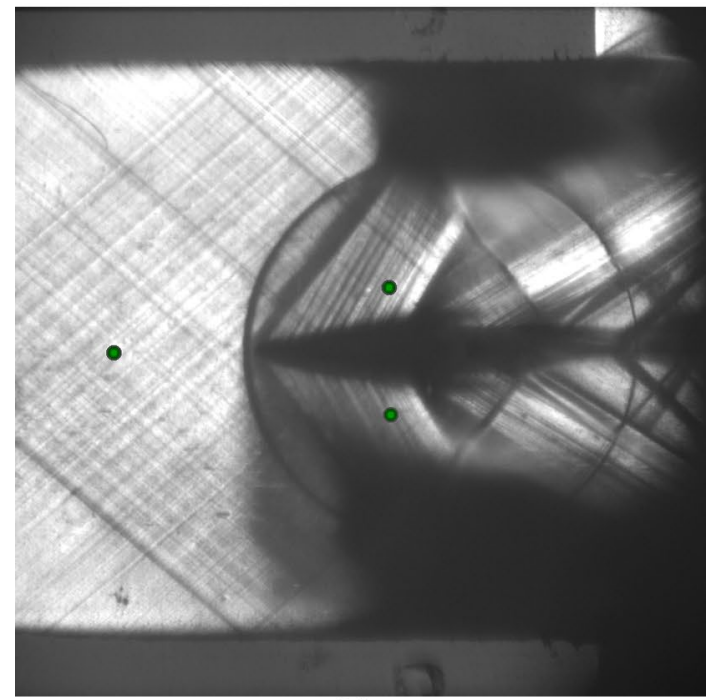

(b)

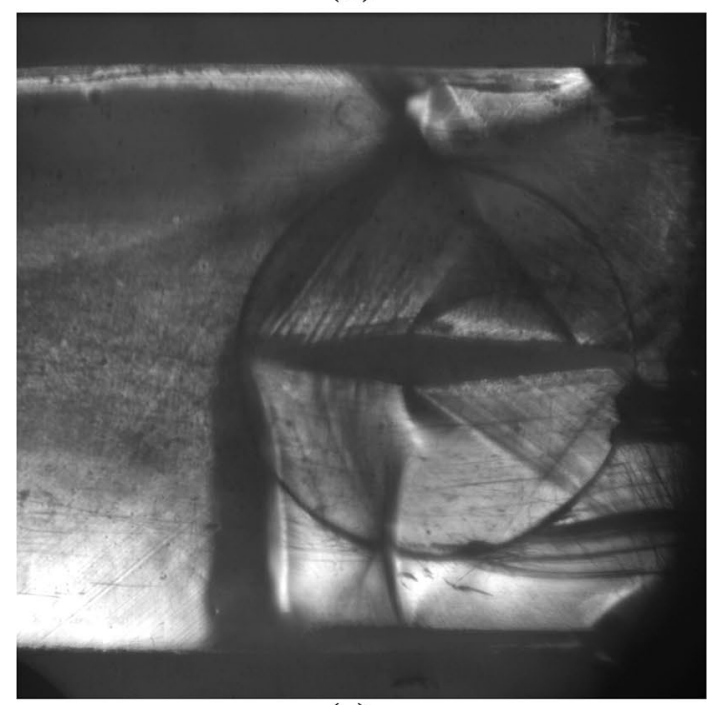

(c) 
and the top portion of Fig. 5c reveal two distinct flow patterns at the boundaries of the constant-area duct, where the two leading edge shocks interact with the wall boundary layer. For the test in configuration $\mathcal{A}$ (Fig. 5a), interaction of the leading edge shocks with the upper and lower walls of the test section results in two reflected shocks, which eventually intersect downstream of the airfoil. For the tests in configuration $\mathcal{B}$ (Fig. 5b, c) instead, flow visualizations reveal that the reflected shocks cross the uniform flow region downstream of the centered expansion fans.

Figure $5 \mathrm{c}$ shows that the interaction of the leading edge shock with the boundary layer on the upper wall of the test section originates a lambda-type reflection. The reflected shock eventually hits the top surface of the airfoil on the expansion ramp. In Fig. 5b, the interaction of the leading edge shocks with the upper and lower walls of the test section is not clearly visible. The shock angle and position suggests, however, that also in these cases a Mach reflection occurred on the upper and lower walls of the wind tunnel.

\subsection{Measurements results}

Experimental results for shock waves deflecting the flow through angles $\vartheta_{\mathrm{B}}<10^{\circ}$ are summarized in Table 2. For each value of the flow deviation angle, the table reports the reservoir, pre-shock and post-shock states measured in five different flow conditions, with the corresponding measurement uncertainties. The table also provides the flow Mach number $M_{\mathrm{A}, \mathrm{th}}$ and fundamental derivative of gasdynamics $\Gamma_{\mathrm{A}, \mathrm{th}}$ in the pre-shock state computed using one-dimensional isentropic theory. Specifically, the values of $\Gamma_{\mathrm{A}, \mathrm{th}}$ and $M_{\mathrm{A}, \mathrm{th}}$ are computed using the SW EoS from the measured values of $P_{\mathrm{R}}, T_{\mathrm{R}}$ and $P_{\mathrm{A}}$, and assuming that both the entropy and total enthalpy are constant from the reservoir $\mathrm{R}$ to the pressure tap 0 upstream of the airfoil, where $P_{\mathrm{A}}$ is measured. In Table 2 and throughout the following discussion, uncertainties on the calculated flow variables are obtained by propagating the uncertainties on the measured $P_{\mathrm{R}}, T_{\mathrm{R}}$, and $P_{\mathrm{A}}$ within the Rankine-Hugoniot jump relations using a standard Monte Carlo method. For all tests, the flow conditions identified as run $i-5$ are characterized by close-to-dilute conditions. In all conditions reported in Table 2, both the compressibility factor $Z$ and the fundamental derivative of gasdynamics $\Gamma$ are less than unity in pre-shock state, which, therefore, lies within the non-ideal compressible-fluid region.

Comparison of the experimental results of Table 2 with the shock wave theory is summarized in the parity plot of Fig. 6. In the plot, the measured pressure ratios $\left(P_{\mathrm{B}} / P_{\mathrm{A}}\right)_{\text {exp }}$, including the corresponding measurements uncertainties, are compared to the computed values $\left(P_{\mathrm{B}} / P_{\mathrm{A}}\right)_{\text {th }}$ resulting from the solution to the Rankine-Hugoniot jump relations, complemented with the SW EoS. The spread of theoretical predictions with respect to the measured values is within $\pm 5 \%$.

The surface of the wind tunnel walls and of the airfoil is machined so that schlieren visualizations of the tested configurations (cf. Fig. 5) clearly display the Mach waves in the diverging portion of the nozzle and around the airfoil. The slope of the Mach waves with respect to the local flow direction provides a direct measurement of the local Mach number, as described in Sect. 2.1. The same technique is applied to the measurement of the shock angle $\beta$. The quality of the schlieren images acquired in flow conditions $2-1,2-2$, and $6-2$ of Table 2 is good enough to allow the detection of Mach waves in the diverging portion of the nozzle and in the flow around the airfoil. The Mach number in the pre- and post-shock states $M_{\mathrm{A}}$ and $M_{\mathrm{B}}$, respectively, and the shock angle $\beta$ measured from the schlieren images of Fig. 5a, b (runs $2-1$ and runs 6-2), and from the schlieren visualization of the test in configuration $\mathcal{A}, \alpha=0^{\circ}, \Gamma_{\mathrm{A}}=0.942$ (runs 2-2) are reported in Table 3, together with the corresponding measurement uncertainties. The table also reports the computed values of the pre-shock flow Mach number $M_{\mathrm{A}, \mathrm{th}}$, of the post-shock Mach number $M_{\mathrm{B}, \mathrm{th}}$ and of the shock angle $\beta_{\text {th }}$. The pre-shock Mach number $M_{\mathrm{A}, \mathrm{th}}$ is calculated from the measured values of $P_{\mathrm{R}}, T_{\mathrm{R}}$ and $P_{\mathrm{A}}$, using the SW EoS and the one-dimensional isentropic theory. The post-shock Mach number $M_{\mathrm{B}, \mathrm{th}}$ and the shock angle $\beta_{\text {th }}$ result from the solution of the Rankine-Hugoniot jump relations complemented with the SW EoS.

In Fig. 7, the measured pre-shock Mach number $M_{\mathrm{A}}$, post-shock Mach number $M_{\mathrm{B}}$, post- to pre-shock Mach number ratio $M_{\mathrm{B}} / M_{\mathrm{A}}$, and shock angle $\beta$ are compared to the ones resulting from one-dimensional isentropic theory and from the Rankine-Hugoniot jump relations, complemented with the SW EoS. For all quantities, the spread of theoretical predictions with respect to the measured values and related uncertainties is well within $\pm 10 \%$.

\subsection{Discussion}

The quasi-one-dimensional theory for isentropic nozzle flows (see e.g. Thompson 1988; Guardone and Vimercati 2016) predicts that, in the NICFD regime, the exhaust Mach number and the exhaust to total pressure ratio depend on the inlet total thermodynamic state. The experimental campaign documented by Spinelli et al. (2018) provided evidence of this dependence, in that flows at different exhaust Mach number and exhaust to total pressure ratios were measured at varying inlet total thermodynamic state. Results reported in Tables 2 and 3 further confirm these findings. As the reservoir state $\mathrm{R}$ approaches more dilute conditions, the ratio of pressure $P_{\mathrm{A}}$ measured at pressure tap 0 to the reservoir pressure $P_{\mathrm{R}}$ decreases. The decrease of the pressure ratio $P_{\mathrm{A}} / P_{\mathrm{R}}$ over each test run is clearly observable in Fig. 8, which reports 


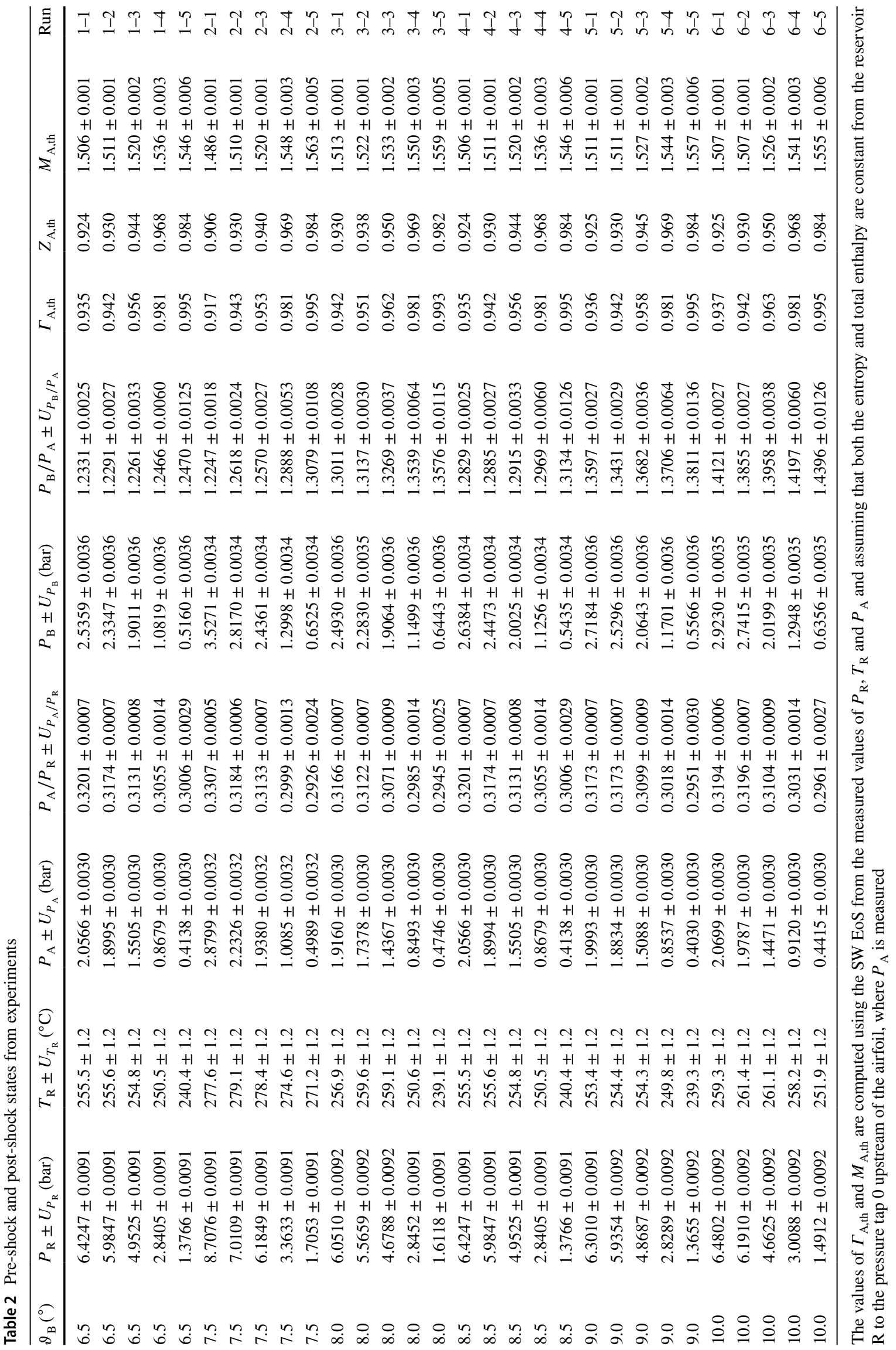




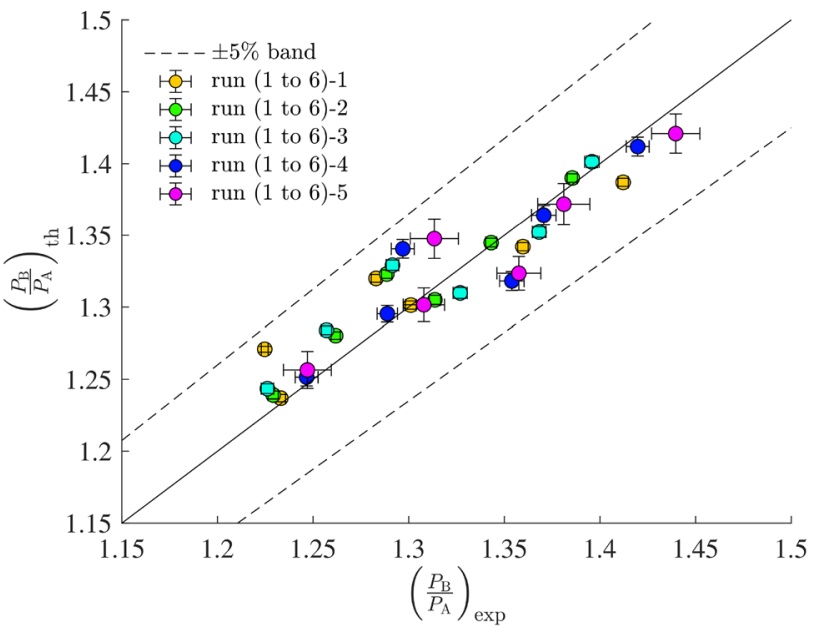

Fig. 6 Parity plot for test results on oblique shock waves. The measured pressure ratio $P_{\mathrm{B}} / P_{\mathrm{A}}$ is compared to the one resulting from the Rankine-Hugoniot jump relations. Flow conditions are given in Table 2

the pressure ratio $P_{\mathrm{A}} / P_{\mathrm{R}}$ in all the test conditions of Table 2, i.e. for all flow deviation angles $\vartheta_{\mathrm{B}}$. Correspondingly, the Mach number $M_{\mathrm{A}}$ measured at pressure tap 0 increases as more dilute conditions are reached (2nd column of Table 3 ). This trend is also predicted by theory in all examined test conditions. Indeed, the pre-shock Mach number $M_{\mathrm{A}, \mathrm{th}}$ monotonically increases from the most non-ideal to the most dilute condition attained in each test, as reported in the 10th column of Table 2. Therefore, measurements carried out in the present experimental campaign confirm that the pre-shock conditions, i.e. the wind-tunnel nozzle exhaust conditions, depend on the reservoir state and change over time within the same run, including pre-shock Mach number $M_{\mathrm{A}}$.

In a perfect gas, the pressure ratio across an oblique shock waves depends uniquely on the shock angle $\beta$, on the upstream Mach number $M_{\mathrm{A}}$ and on the value of the specific heat ratio $\gamma$, which is constant for a given fluid regardless of its thermodynamic state. In particular, the ratio $P_{\mathrm{B}} / P_{\mathrm{A}}$ increases as the pre-shock Mach number $M_{\mathrm{A}}$ increases. On the contrary, in non-ideal flows a dependence of the post-shock state on the upstream (pre-shock) thermodynamic state is expected. The value of the pressure ratio across the shock wave results as a combination of the effect of non-ideal behavior of the wind tunnel already observed and quantified by Spinelli et al. (2018) and of the non-ideality of the shock adiabat, which is the focus of the present analysis. Wind tunnel non-ideality results in the increase of the Mach number, and hence of the pressure ratio $P_{\mathrm{B}} / P_{\mathrm{A}}$ across the shock (Fig. 9), as discussed above. In test runs 5-1 and 5-2 the non-ideality of the shock adiabat is apparent. Comparing the two shock waves in runs 5-1 and 5-2, namely the most non-ideal conditions of test 5 , one finds that the computed pre-shock Mach number is the same in both runs 5-1 and 5-2, namely $M=1.511 \pm 0.001$, whereby the pressure ratios across the shock differ, with $P_{\mathrm{B}} / P_{\mathrm{A}}=1.3597 \pm 0.0027$ for runs $5-1$ and $P_{\mathrm{B}} / P_{\mathrm{A}}=1.3431 \pm 0.0029$ for runs 5-2. The same occurs for the non-ideal conditions 6-1 and 6-2, with $M=1.511 \pm 0.001$ in both runs but $P_{\mathrm{B}} / P_{\mathrm{A}}=1.4121 \pm 0.0027$ for runs $6-1$ and $P_{\mathrm{B}} / P_{\mathrm{A}}=1.3855 \pm 0.0027$ for runs $6-2$. Data reported in Table 3 also suggest that, in each test run, the shock angle decreases and the post- to pre-shock Mach number ratio $M_{\mathrm{B}} / M_{\mathrm{A}}$ decreases as more dilute conditions are approached. Note that the due to the overlap of the uncertainties for Mach number and shock angle $\beta$ measurements reported in Table 3, no clear indications with respect to the aforementioned nonideal behavior can be inferred from these quantities.

\section{Conclusions}

Oblique shock waves were observed experimentally in the non-ideal compressible-fluid regime for siloxane fluid MDM. Experiments were carried out in the TROVA blowdown wind tunnel of Politecnico di Milano. Oblique shock waves occurring in a uniform supersonic flow at Mach number 1.5 were studied for a set of flow deviation angles through independent measurements of temperature, pressure and Mach number. For all oblique shock configurations observed, pressure and Mach number measurements agree fairly well with the inviscid shock wave theory,

Table 3 Mach number and shock angle measurements in flow conditions 2-1, 2-2, and 6-2 of Table 2

\begin{tabular}{|c|c|c|c|c|c|c|}
\hline & \multicolumn{2}{|c|}{$\vartheta_{\mathrm{B}}=7.5^{\circ}$, runs $2-1$} & \multicolumn{2}{|c|}{$\vartheta_{\mathrm{B}}=7.5^{\circ}$, runs $2-2$} & \multicolumn{2}{|c|}{$\vartheta_{\mathrm{B}}=10^{\circ}$, runs $6-2$} \\
\hline & Experiment & Theory & Experiment & Theory & Experiment & Theory \\
\hline$M_{\mathrm{A}} \pm U_{M_{\mathrm{A}}}$ & $1.544 \pm 0.041$ & $1.486 \pm 0.001$ & $1.601 \pm 0.040$ & $1.510 \pm 0.001$ & $1.527 \pm 0.032$ & $1.507 \pm 0.001$ \\
\hline$M_{\mathrm{B}} \pm U_{M_{\mathrm{B}}}$ & $1.397 \pm 0.027$ & $1.334 \pm 0.001$ & $1.362 \pm 0.029$ & $1.350 \pm 0.001$ & $1.308 \pm 0.016$ & $1.287 \pm 0.002$ \\
\hline$M_{\mathrm{B}} / M_{\mathrm{A}} \pm U_{M_{\mathrm{B}}} / M_{\mathrm{A}}$ & $0.905 \pm 0.030$ & $0.898 \pm 0.001$ & $0.851 \pm 0.028$ & $0.894 \pm 0.001$ & $0.857 \pm 0.021$ & $0.854 \pm 0.001$ \\
\hline$\beta \pm U_{\beta}\left({ }^{\circ}\right)$ & $52.00 \pm 0.40$ & $49.27 \pm 0.04$ & $51.34 \pm 0.49$ & $48.46 \pm 0.05$ & $51.56 \pm 0.39$ & $51.35 \pm 0.06$ \\
\hline
\end{tabular}

Computed values of the pre-shock flow Mach number $M_{\mathrm{A}, \mathrm{th}}$, of the post-shock Mach number $M_{\mathrm{B}, \mathrm{th}}$ and of the shock angle $\beta_{\mathrm{th}}$ in flow conditions $2-1,2-2$, and $6-2$ of Table 2 
Fig. 7 Parity plot for test results on oblique shock waves. The measured pre-shock Mach number $M_{\mathrm{A}}$, post-shock Mach number $M_{\mathrm{B}}$, post- to pre-shock Mach number ratio $M_{\mathrm{B}} / M_{\mathrm{A}}$, and shock angle $\beta$ are compared to the ones resulting from onedimensional isentropic theory and from the Rankine-Hugoniot jump relations, complemented with the SW EoS. Data reported in the plots are provided in Table 2 and in Table 3
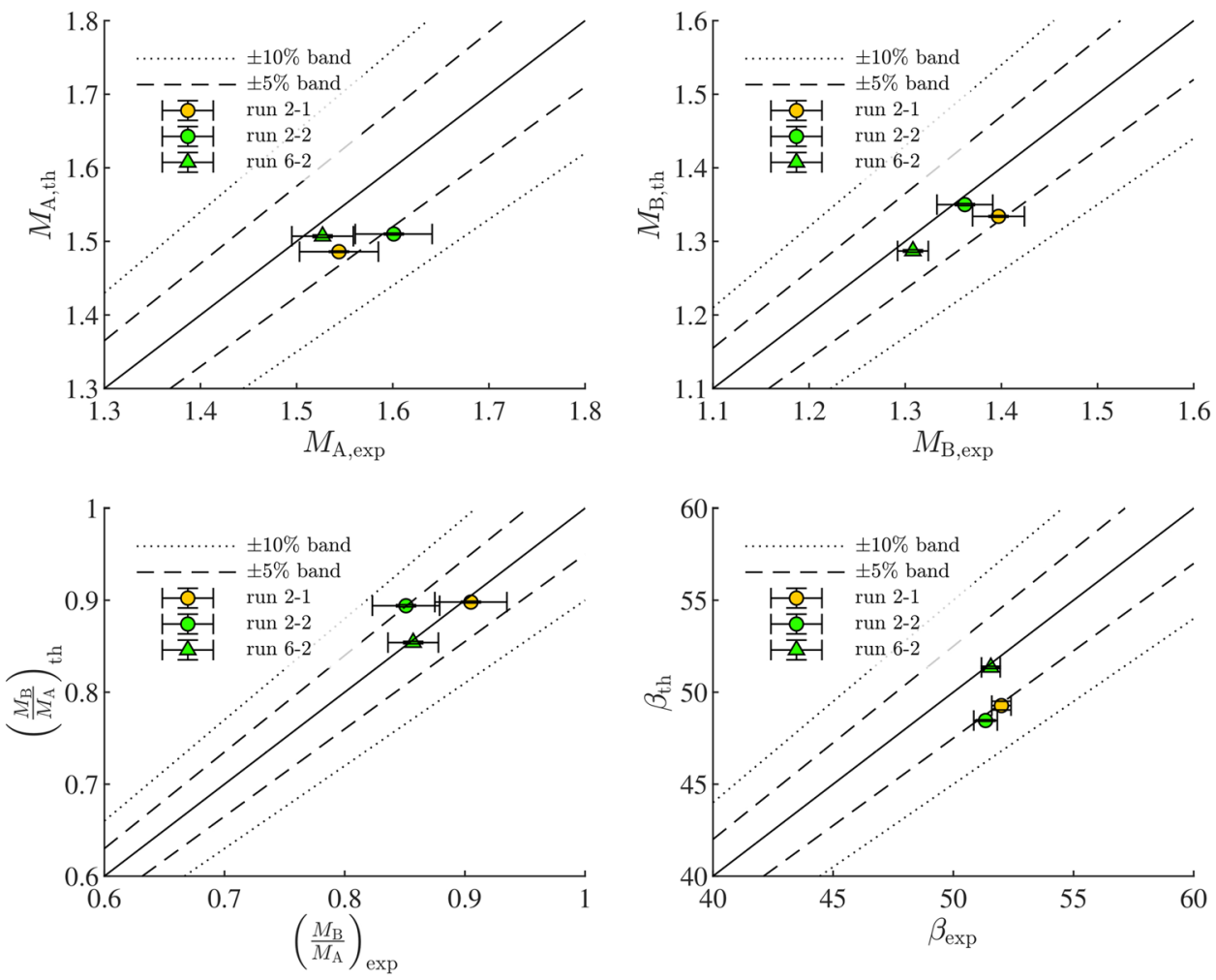

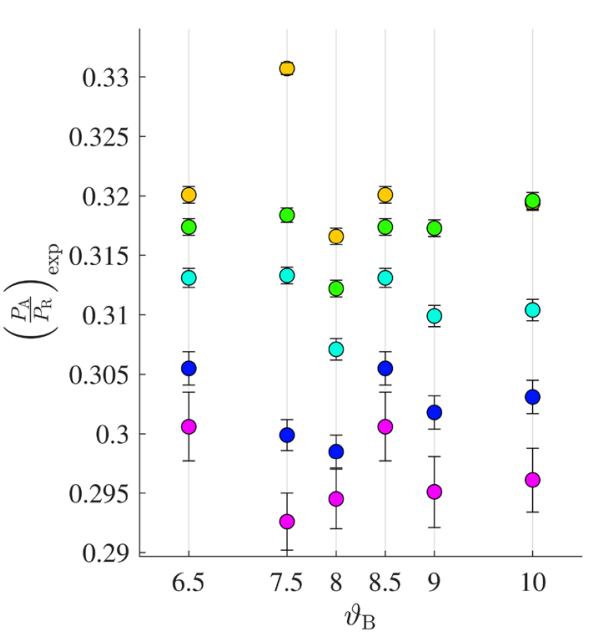

Fig. 8 Measured pressure ratio $P_{\mathrm{A}} / P_{\mathrm{R}}$ at varying flow deviation angle $\vartheta_{B}$. Flow conditions are given in Table 2

complemented with an equation of state accounting for non-ideal flow behavior. Reference experimental data are provided for oblique shocks realizing flow turning by six angles in the range $\vartheta_{B}=6.5^{\circ}-10^{\circ}$, for upstream thermodynamic states featuring reduced stagnation temperatures and pressures ranging from 0.928 to 0.979 and from 0.200 to 0.615 , respectively. The corresponding values of the fundamental derivative of gasdynamics range from 0.917 to 0.995 according to state-of-the-art thermodynamic models, which place the current experiment within the non-ideal regime of

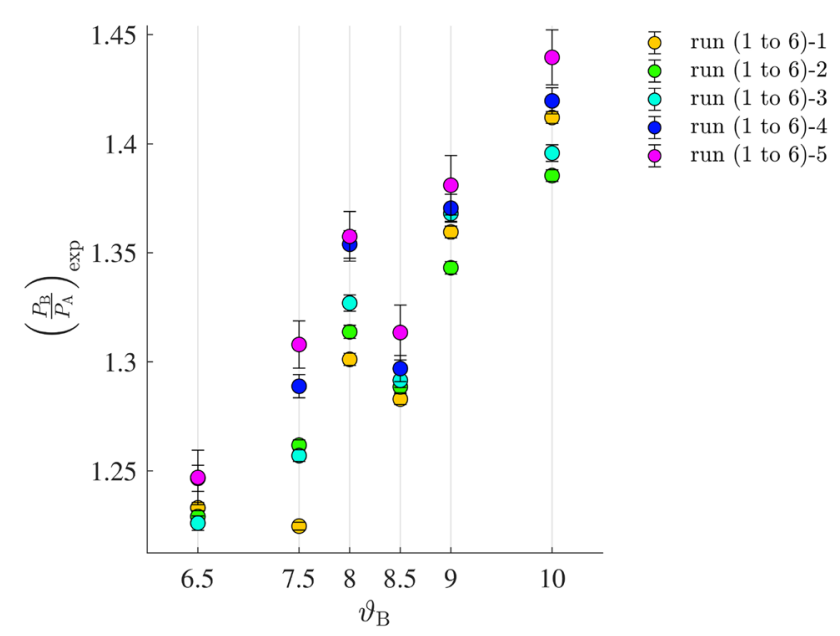

Fig. 9 Measured pressure ratio $P_{\mathrm{B}} / P_{\mathrm{A}}$ at varying flow deviation angle $\vartheta_{B}$. Flow conditions are given in Table 2

$\Gamma<1$. In addition to the non-ideal effects on wind tunnel operation observed in Spinelli et al. (2018), which causes the wind tunnel Mach number to increase as dilute conditions are attained, experimental results confirm that the post-shock states in non-ideal flows depend on the local and total flow variables in the pre-shock state, as predicted by the shock wave theory for non-ideal flows.

Acknowledgements The research is funded by the European Research Council under ERC Consolidator Grant 2013 No. 617603 NSHOCK. 
The authors wish to thank prof. Luigi Vigevano for helpful discussion regarding the design of the experiment and Camilla Conti, Simone Gallarini and Davide Vimercati for their help during experimental trials and data processing.

Open Access This article is distributed under the terms of the Creative Commons Attribution 4.0 International License (http://creativeco mmons.org/licenses/by/4.0/), which permits unrestricted use, distribution, and reproduction in any medium, provided you give appropriate credit to the original author(s) and the source, provide a link to the Creative Commons license, and indicate if changes were made.

\section{References}

Angelino G (1969) Real gas effects in carbon dioxide cycles. ASME J Eng Power 10(13):272

Angelino G, Invernizzi C, Macchi E (1991) Organic working fluid optimization for space power cycles: chap 16. In: Angelino G, DeLuca L, Sirignano WA (eds) Modern research topics in aerospace propulsion. Springer, New York

Antonini C, Persico G, Rowe L (2008) Prediction of the dynamic response of complex transmission line systems for unsteady pressure measurements. Meas Sci Technol 19(12):125,401

Colonna P, Guardone A (2006) Molecular Interpretation of nonclassical gasdynamics of dense vapors under the van der Waals model. Phys Fluids 18(5):56,101-56,114

Colonna P, Guardone A, Nannan RN (2007) Siloxanes: a new class of candidate Bethe-Zel'dovich-Thompson fluids. Phys Fluids 19:086102

Colonna P, Harinck J, Rebay S, Guardone A (2008) Real-gas effects in organic Rankine cycle turbine nozzles. J Propuls Power 24(2):282-294

Colonna P, Casati E, Trapp C, Mathijssen T, Larjola J, Turunen-Saaresti T, Uusitalo A (2015) Organic Rankine cycle power systems: from the concept to current technology, applications and an outlook to the future. ASME J Eng Gas Turbines Power 137(100):801

Cozzi F, Spinelli A, Carmine M, Cheli R, Zocca M, Guardone A (2015) Evidence of complex flow structures in a converging-diverging nozzle caused by a recessed step at the nozzle throat. In: 45th AIAA fluid dynamics conference

Cramer MS, Best LM (1991) Steady, isentropic flows of dense gases. Phys Fluids A Fluid Dyn 3(4):219

Denton JD (1993) Loss mechanisms in turbomachines. In: International gas turbine and aeroengine congress and exposition, pp 93-GT-435

Feher EG (1968) The supercritical thermodynamic power cycle. Energy Convers 8:85-90

Guardone A, Vimercati D (2016) Exact solutions to non-classical steady nozzle flows of Bethe-Zel'dovich-Thompson fluids. J Fluid Mech 800:278-306

Guardone A, Zamfirescu C, Colonna P (2009) Maximum intensity of rarefaction shock waves for dense gases. J Fluid Mech 642:127

Guardone A, Spinelli A, Dossena V (2013) Influence of molecular complexity on nozzle design for an organic vapor wind tunnel. ASME J Eng Gas Turbines Power 135(042):307
Hung PC, Irwin G, Kee R, McLoone S (2005) Difference equation approach to two-thermocouples sensor characterization in constant velocity flow environment. Rev Sci Instrum 76:024902

Keulen L, Gallarini S, Landolina C, Spinelli A, Iora P, Invernizzi C, Lietti L, Guardone A (2018) Thermal stability of hexamethyldisiloxane and octamethyltrisiloxane. Energy 165:868-876. https ://doi.org/10.1016/j.energy.2018.08.057

Kluwick A (2017) Non-ideal compressible fluid dynamics: a challenge for theory. J Phys Conf Ser 821(1):012,001

Lambrakis KC, Thompson PA (1972) Existence of real fluids with a negative fundamental derivative $\Gamma$. Phys Fluids 15(5):933-935

Larjola J (1995) Electricity from industrial waste heat using high-speed Organic Rankine Cycle (ORC). Int J Prod Econ 41:227-235

Lo RC, Tsai WH (1995) Gray-scale Hough trasform for thick line detection in grey-scale images. Pattern Recognit 28:647-661

Macchi E, Astolfi M (eds) (2016) Organic Rankine Cycle (ORC) power systems technologies and applications, 1st edn. Amsterdam, Elsevier

Reverchon E, Pallado P (1996) Hydrodynamic modeling of the RESS process. J Supercrit Fluids 9(4):216-221

Schnerr GH, Leidner P (1991) Diabatic supersonic flows of dense gases. Phys Fluids A Fluid Dyn 3(10): 2445

Spinelli A, Pini M, Dossena V, Gaetani P, Casella F (2013) Design, simulation, and construction of a test rig for organic vapours. ASME J Eng Gas Turbines Power 135(042):303

Spinelli A, Cozzi F, Cammi G, Zocca M, Gaetani P, Dossena V, Guardone A (2017) Preliminary characterization of an expanding flow of siloxane vapor MDM. J Phys Conf Ser 821(1):012,022

Spinelli A, Cammi G, Gallarini S, Zocca M, Cozzi F, Gaetani P, Dossena V, Guardone A (2018) Experimental evidence of non-ideal compressible effects in expanding flow of a high molecular complexity vapor. Exp Fluids 59(8):126

Subramaniam B, Rajewski RA, Snavely K (1997) Pharmaceutical processing with supercritical carbon dioxide. J Pharm Sci 86(8):885-890

Sun XY, Wang TJ, Wang ZW, Jin Y (2002) The characteristics of coherent structures in the rapid expansion flow of the supercritical carbon dioxide. J Supercrit Fluids 24(3):231-237

Thol M, Dubberke FH, Baumhögger E, Vrabec J, Span R (2017) Speed of sound measurements and fundamental equations of state for octamethyltrisiloxane and decamethyltetrasiloxane. J Chem Eng Data 62:2633-2648

Thompson PA (1971) A fundamental derivative in gasdynamics. Phys Fluids 14(9):1843-1849

Thompson PA (1988) Compressilbe-fluid dynamics. McGraw-Hill, New York

Vimercati D, Gori G, Guardone A (2018) Non-ideal oblique shock waves. J Fluid Mech 847:266-285. https://doi.org/10.1017/ jfm.2018.328

Zamfirescu C, Guardone A, Colonna P (2008) Admissibility region for rarefaction shock waves in dense gases. J Fluid Mech 599:363-381

Publisher's Note Springer Nature remains neutral with regard to jurisdictional claims in published maps and institutional affiliations.

\section{Affiliations}

\section{Marta Zocca ${ }^{1} \cdot$ Alberto Guardone $^{1} \mathbb{D} \cdot$ Giorgia Cammi $^{2} \cdot$ Fabio Cozzi $^{2} \cdot$ Andrea Spinelli $^{2}$}

1 Department of Aerospace Science and Technology, Politecnico di Milano, Via La Masa 34, 21056 Milan, Italy
2 Energy Department, Politecnico di Milano, Via

Lambruschini 4, 21056 Milan, Italy 